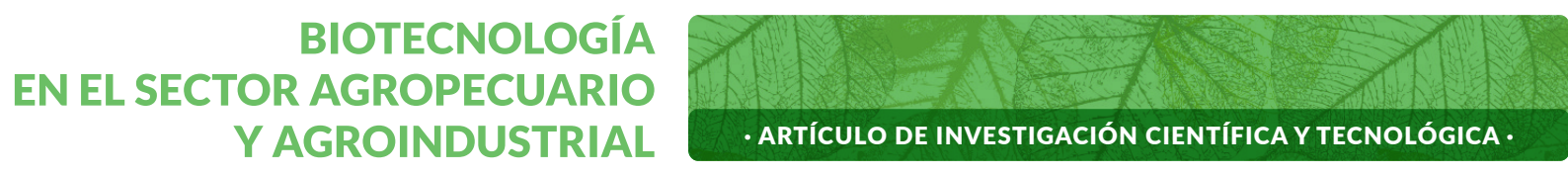

Vol. 16 No 2 · Julio - Diciembre 2018 • ISSN - 1692-3561 · ISSN-e 1909-9959 · doi: http://dx.doi.org/10.18684/bsaa.v16n2.99

\title{
Estado de los recursos naturales en el parque nacional molino de flores Netzahualcóyotl
}

\author{
Natural resources status in the molino \\ de flores Netzahualcóyotl national park
}

\section{Estado da dos recursos naturais no parque nacional molino de flores Netzahualcóyotl}

\author{
ALEJANDRO HERRERA-LLAMPALLAS ${ }^{1}$, ARIANA LUQUE-DELGADILLO', \\ GUILLERMO RAMÍREZ-GARCÍA ${ }^{2}$, ANASTASIO ESPEJEL-GARCÍA ${ }^{3}$, \\ ANTONIO VÁZQUEZ-ALARCÓN , JESÚS D. GÓMEZ-DÍAZ4, \\ ALEJANDRO I. MONTERROSO-RIVAS ${ }^{4}$
}

\section{RESUMEN}

El Parque Nacional Molino de Flores Netzahualcóyotl es un área natural protegida que se encuentra en el municipio de Texcoco y es administrada por el Ayuntamiento. El área carece de un plan de manejo o alguna otra guía o documento de planeación que marque pauta en el manejo y conservación de sus recursos naturales. Con el

Recibido para evaluación: 28 de Febrero de 2018.

Aprobado para publicación: 25 de Julio de 2018.

1 Departamento de Suelos, Centro de Investigación en Recursos Naturales y Medio Ambiente - CIRENAM. Universidad Autónoma Chapingo. Ingeniero en Recursos Naturales. Texcoco, México.

2 Centro Regional Universitario del Noroeste, Centro de Investigación en Recursos Naturales y Medio Ambiente - CIRENAM Universidad Autónoma Chapingo. Doctor en Geografía. Ciudad Obregón, México.

3 Departamento de Ingeniería Agroindustrial, Universidad Autónoma Chapingo. Doctor. Texcoco, México.

4 Departamento de Suelos, Centro de Investigación en Recursos Naturales y Medio Ambiente - CIRENAM. Universidad Autónoma Chapingo. Doctor en Edafología. Texcoco, México. 
objetivo de hacer un inventario y conocer su estado actual de conservación se analizó información obtenida en campo de 51 sitios de muestreo en sus 45 hectáreas de extensión. El método consistió en delimitar el parque y realizar un inventario y diagnóstico de los recursos naturales. Se tomaron datos sobre el estado actual del suelo (18 parámetros), vegetación (36 parámetros en 5 estratos) y agua (4 puntos, 16 muestras y 9 parámetros cada una). La información revela los problemas presentes y en gran mayoría con tendencias de deterioro que padece el parque: disminución de cobertura vegetal, contaminación de suelo y agua, descargas de aguas residuales contaminadas en el río Coxcacuaco, altas tasas por erosión hídrica, deforestación y extracción de recursos forestales. Los resultados permiten conocer las principales causas de la degradación de los recursos y a su vez las áreas de oportunidad para propiciar su rehabilitación, correcto manejo y administración del parque.

\section{ABSTRACT}

"Molino de Flores Netzahualcoyotl" National Park is a protected area located in Texcoco, Mexico and is managed by the municipality. The area lacks a management plan or any other guide or planning document that sets standards in management and conservation of its natural resources. The main goal was to make a natural resources inventory and current conservation status, thus information was obtained from 51 sampling sites in its 45 hectares of extension. The method was to delimit the park and carry out an inventory and diagnosis of natural resources. Data collected on the current state of the soil (18 parameters), vegetation (36 parameters in 5 strata) and water (4 points, 16 samples and 9 parameters each one). Information exposes the present problems and tendencies of deterioration in the National Park: decrease of vegetal cover, soil and water pollution, discharges of contaminated residual water in the Coxcacuaco river, high rates in soils by water erosion, deforestation and illegal extraction of forest resources. The results allow to know the main causes of the degradation of the natural resources and at the same time the areas of opportunity to propitiate their rehabilitation, correct management and administration of the park.

\section{RESUMO}

O Parque Nacional Molino de Flores Netzahualcoyotl é uma área natural protegida que está localizada no município de Texcoco e é administrada pela Câmara Municipal. A área não possui um plano de gerenciamento ou qualquer outro guia ou documento de planejamento que estabeleça diretrizes para o gerenciamento e conservação de seus recursos naturais. Para fazer um inventário e conhecer o estado atual de conservação, as informações obtidas no campo foram analisadas a partir de 51 locais de amostragem em seus 45 hectares. 0 método consistiu em delimitar o parque e realizar um inventário e diagnóstico dos recursos naturais. Os dados foram coletados sobre o estado atual do solo (18 parâmetros), vegetação (36 parâmetros em 5 estratos) e água (4 pontos, 16 amostras e 9 parâmetros cada). A informação revela os problemas presentes e em grande maioria com tendências de deterioração que o parque sofre: diminuição da cobertura vegetal, contaminação

\section{PALABRAS CLAVE:}

Estado de conservación, Diagnóstico, Zonificación, Inventario, Gestión ambiental.

\section{KEYWORDS:}

Conservation status, Diagnosis, Zoning, Inventory, Environmental management.

\section{PALAVRAS-CHAVE:}

Estado de conservação, Diagnóstico, Zoneamento, Inventário, Gestão ambiental. 
do solo e água, descarga de água residual contaminada no rio Coxcacuaco, altas taxas por erosão da água, desmatamento e extração de recursos florestais. Os resultados permitem conhecer as principais causas da degradação dos recursos e, ao mesmo tempo, as áreas de oportunidade para propiciar sua reabilitação, gerenciamento correto e administração do parque.

\section{INTRODUCCIÓN}

Reconocer el estado actual de los recursos naturales es fundamental para proponer la estrategia de manejo y preservación de los parques en el tiempo. El inventario de los recursos forestales es importante para la toma de decisiones y el desarrollo de un plan de manejo [1]. Los parques nacionales deben cumplir con características específicas y estar bajo acciones concretas de manejo y cuidado [2]. En México, las reglas de funcionamiento de un parque se estipulan en la Ley General de Equilibrio Ecológico y Protección al Ambiente [3]. El Parque Nacional Molino de Flores Netzahualcóyotl (PNMFN) tiene la categoría de "Parque Nacional" desde el 5 de noviembre de 1937, cuando se decretó por el presidente Lázaro Cárdenas. Esta categoría puede ser equivalente a la III de la UICN, conservación de los rasgos naturales, monumento natural o monumento histórico. Los parques nacionales se definen como representaciones biogeográficas, a nivel nacional, de uno o más ecosistemas [4] que signifiquen importancia por su belleza escénica, su valor científico, educativo, de recreo, su valor histórico, por la existencia de flora y fauna, por su aptitud para el desarrollo del turismo, o bien por otras razones análogas de interés general.

Desde enero de 1997 el PNMFN está bajo la administración del municipio de Texcoco desde dos dependencias: la Dirección de ecología, (que se encarga del personal administrativo, los planes de trabajo anuales, reforestación y actividades generales) y la Dirección de regulación comercial, que se encarga del cobro de cuotas (estacionamiento, pagos semanales y anuales de los comerciantes).

Existen serios problemas ambientales, ocasionados por el exceso de basura producida por los usuarios, vertido de aguas negras al río, contaminación del aire por las minas de arena, extracción de la vegetación e incendios [5], aunado a la falta de presupuesto y posible indiferencia por parte de las autoridades mu- nicipales, que incrementa la vulnerabilidad del equilibrio ecológico del sitio.

Veinte años después de ser administrado por el municipio, surge el interés de evaluar la degradación y el estado actual, que implica realizar una comparación entre el estatus en un determinado momento, con un estado previo que es identificado como línea base o estado de referencia [6], que da relevancia a la recolección de información y la caracterización de las masas forestales y áreas naturales, tanto para conocer el avance de dicha degradación [7] como para conocer el resultado de posibles medidas a ser aplicadas.

El PNMFN destaca por su relevancia histórica, cultural y de esparcimiento para la comunidad local y turistas [8]. El objetivo del presente fue hacer un inventario y diagnóstico del suelos, vegetación y río en el PNMFN, con el propósito de conocer su estado actual de conservación y establecer las bases necesarias para elaborar una propuesta de manejo integral de sus recursos naturales. Lo anterior busca integrar la funcionalidad ecológica, así como la administrativa, con el fin de mejorar las practicas actuales y por consiguiente el estado de los recursos naturales. La motivación para mantener áreas protegidas es cumplir con los objetivos de preservar en su condición original los recursos y ecosistemas para el uso y disfrute de las generaciones futuras y actuales [9].

La presente investigación pretende reconocer el estado que guardan los recursos naturales del área para comprender los procesos dominantes que se presentan en el área, como se ha sugerido previamente [10].

\section{MÉTODO}

\section{Área de Estudio}

El PNMFN se localiza al oriente del estado de México, en el municipio de Texcoco con una superficie de $45,6 \mathrm{~h}$. Presenta cambio altitudinal de $40 \mathrm{~m}$, al pasar de $2290 \mathrm{msnm}$ y hasta los $2330 \mathrm{msnm}$. Se encuentra rodeado de lomeríos y dentro del área se pueden encontrar pendientes de $4,5 \%$ a más de $70 \%$ en la ribera del rio [5]. El clima dominante es templado con temperatura media anual de $14^{\circ} \mathrm{C}$ y precipitación de 620 $\mathrm{mm}$. Los vientos provienen del noroeste durante la estación seca de invierno y los del noreste en la estación de verano, manteniendo velocidad aproximada de 10 
$\mathrm{km} / \mathrm{h}$. El rio que lo atraviesa casi por la mitad se llama Coxcacuaco y lo hace de oriente a poniente, teniendo como abastecimiento principal los manantiales de San Francisco, San Juan Totolapa y el manantial de la Joya.

\section{Zonificación}

Dada la imprecisión en el lindero del parque se delimitó el perímetro de este. Se hicieron recorridos a pie y se obtuvieron coordenadas del polígono (en metros según UTM-14) con ayuda del sistema de posicionamiento Magellan Mobilmapper 6 [11, 12]. Después, se entrevistó al personal administrativo para corroborar la información y conocer su opinión respecto a los usos de suelos dominantes [13] y sobre las áreas que presentan mayor afluencia de visitantes. Además, se estimó la densidad de la vegetación y la pendiente del terreno como lo sugiere CONAFOR [14]. A partir de lo anterior y utilizando una imagen de satélite reciente como base (Google Earth, 2017) se delimitaron 14 zonas que fungen como áreas de manejo o gestión ambiental (Figura 1).

\section{Estado Actual de la Conservación}

Se obtuvo a partir del análisis del estado de los suelos, la vegetación y el río. Cabe señalar que no se abordó la fauna. El proceso se describe a continuación:

Suelos. Se realizó un muestreo a partir de una rejilla de puntos estratificados $(20 \times 20 \mathrm{~m})$ tomando como base el mapa de zonificación generado. Para las 14 zonas se determinaron 51 sitios de muestreo. Se consideró una profundidad de $30 \mathrm{~cm}$ para la obtención de una muestra por cada sitio y se determinaron y registraron los siguientes parámetros: color, textura, pedregosidad, estructura, consistencia (en seco y en húmedo), pegajosidad, plasticidad, materia orgánica, carbonatos, permeabilidad, tipo de drenaje, $\mathrm{pH}$, cantidad y tamaño de poros y raíces, la presencia de micro fauna en el suelo y la profundidad aproximada de cada punto de muestreo, según lo planteado por especialistas en el área [15].

Figura 1. Zonificación y estado actual de conservación en el Parque Nacional Molino de Flores Netzahualcóyotl.

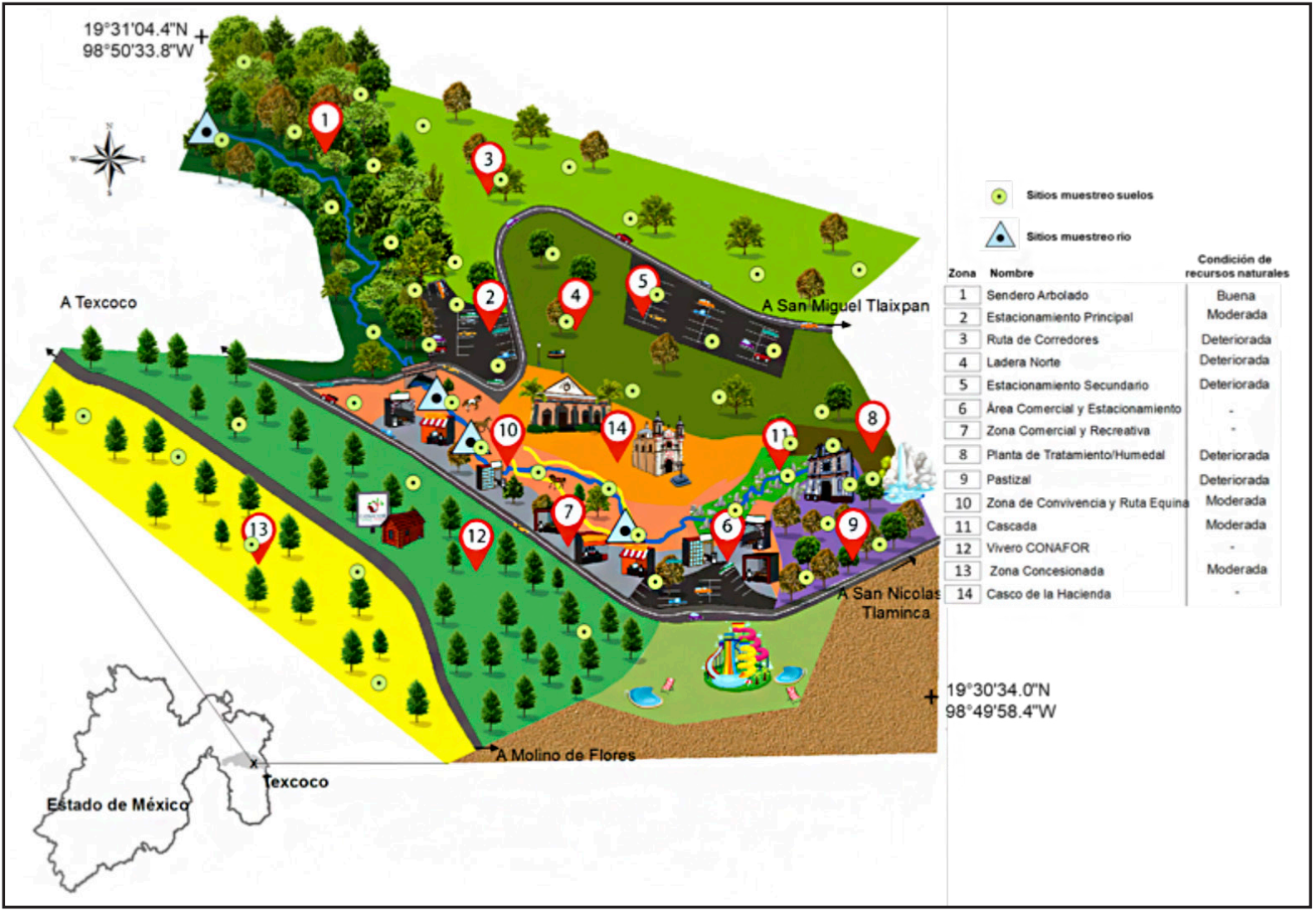


Además, se registró evidencia de degradación del suelo en un área de $400 \mathrm{~m}^{2}$ trazada a partir de cuatro transectos de $10 \mathrm{~m}$ cada uno medidos a partir del centro del sitio (punto donde se realizó el muestreo del suelo), tal como se recomienda en el Inventario Nacional Forestal y de Suelos y estudios de suelos [16, 17].

Vegetación. La vegetación se evaluó en cinco grupos o estratos: vegetación menor, sotobosque, repoblado, arbolado y material leñoso caído. Se determinó la siguiente superficie para la medición de cada estrato: vegetación menor $\left(1 \mathrm{~m}^{2}\right)$, material leñoso (transecto de $20 \mathrm{~m}$ ) y vegetación menor, sotobosque, repoblado y arbolado $\left(400 \mathrm{~m}^{2}\right)$, con el fin de abarcar la mayor cantidad posible de individuos y características presentes en el PNMFN.

Para cada grupo se obtuvieron mediciones sobre: vegetación menor (porcentajes de cobertura ocupada por vegetación como: gramíneas (pastizales), helechos, musgos y líquenes; porcentaje de la superficie cubierto por hojas, suelo desnudo, rocas, gravas y piedras, y otros elementos que ocupen un determinado espacio en el área de medición); sotobosque (especie dominante, forma de vida, porcentaje de cobertura, frecuencia por alturas, vigor de cada individuo, daño presente y porcentaje de daño); repoblado (especie dominante, forma de vida, porcentaje de cobertura, frecuencia por alturas, vigor de cada individuo, daño presente y porcentaje de daño); arbolado (nombre científico, nombre común, forma de vida, forma de fuste, condición, diámetro normal (a $1,30 \mathrm{~m}$ ), altura total, ángulo de inclinación> a $30^{\circ}$, vigor, diámetro basal (a $30 \mathrm{~cm}$ ), cobertura del dosel, daño y dominancia); material leñoso caído (material de 1-10 h; material de $100 \mathrm{~h}$; material de 1000 h; diámetro $(\mathrm{cm})$, porcentaje de cobertura y grado de putrefacción) y arbustos y pastos (altura).

Evaluación del estado actual del parque. Se siguió un método de ponderación multicriterio [18] que consideró 17 variables y siete clases. Para cada variable y siguiendo juicio experto se asignó valor de importancia ponderada para ser integrado después en cinco clases finales. De acuerdo con el valor final unidimensional (de 0,5 a 5,5 ) se clasificaron como: excelente $(0,5$ a 1,5), buena (1,6 a 2,5), moderada (2,6 a 3,5), deteriorada $(3,6$ a 4,5$)$ y muy deteriorada $(4,6$ a 5,5$)$. Cabe subrayar que se excluyeron las áreas destinadas a uso comercial (zonas 6 y 7 ) y vivero forestal (zona 12), dado que no cuentan con la totalidad de resulta- dos (suelo y vegetación) por ser de uso intensivo (suelos cubiertos de cemento, poco arbolado).

Evaluación del estado actual del río. Se realizó la identificación de los factores constitutivos del rio, los cuales se clasificaron en cuatro ambientes: inmediato, generativo, degenerativo y esporádico. Se investigó si su ambiente es favorable [19], realizando muestreos sobre cuatro distintos puntos del río Coxcacuaco, uno de estos afuera y antes de ingresar al PNMFN con el fin de tener una referencia de calidad del agua que llega al parque. Para todos los sitios se realizaron cuatro muestreos (febrero 2016), dos el mismo día; uno en la mañana y otro en la tarde; y los otros dos tres días después también en ambos turnos. Se obtuvieron 16 muestras en total y a cada una se realizaron análisis en el laboratorio sobre; dureza, $\mathrm{pH}$, conductividad eléctrica, solidos totales, solidos solubles, turbidez, alcalinidad, cloruros y demanda bioquímica de oxígeno (DBO) considerando el trabajo de especialistas $[20,21]$.

\section{RESULTADOS}

Se distinguen 14 zonas por diferencias en intensidad de uso, densidad de vegetación, pendiente del terreno y uso actual de suelo. Cabe señalar que la investigación en un sitio generalmente comienza por estudios descriptivos [22]. Así, las zonas obtenidas pueden ser consideradas zonas de manejo o zonas de gestión del parque. Fueron asignados nombres a las zonas de acuerdo con el uso principal del suelo (Figura 1).

Zona 1: Sendero Arbolado. Cubre una superficie de 5,5 ha donde los suelos tienen profundidades de $0,35 \mathrm{~m}$ a más de $1,50 \mathrm{~m}$. La estructura del suelo que predomina es moderadamente desarrollada y la pedregosidad va de $0 \%$ a $10 \%$ aproximadamente, con una baja incidencia de afloramientos rocosos. En el sendero hay baja concentración de raíces y poros en el suelo, con diámetros que van de $1 \mathrm{~mm}$ a $5 \mathrm{~mm}$ aproximadamente en ambos casos. Las pruebas realizadas mostraron alto contenido de materia orgánica. La zona se clasifica con "alta" erosión ya que se encontraron canalillos de hasta $8 \mathrm{~m}$ de distancia y $15 \mathrm{~cm}$ de profundidad, así como también un par de cárcavas de entre 4 y $8 \mathrm{~m}$ de largo por $80 \mathrm{~cm}$ a $1,5 \mathrm{~m}$ de profundidad. Con relación a la cobertura vegetal, se encuentra vegetación riparia en la zona más cercana al río. Después, hacia el norte, los visitantes han trazado con el 
paso de los años un sendero alrededor del cual se encuentran los árboles más viejos y altos de todo el PNMFN; entre ellos eucaliptos (Eucaliptus camaldulensis Dehnh y Eucaliptus globulus Labill), fresnos (Fraxinus udhei (Wenz.) Lingelsh), cedro blanco (Cupressus lindleyi Klotzsch ex Endl.), casuarinas (Casuarina equisetifolia L.), pirúl (Schinus molle L.) y algunos pinos (Pinus montezumae Lamb.). La cobertura es baja en el estrato del sotobosque, las principales especies presentes son la uña de gato (Mimosa aculeaticarpa Hort.), trompetilla (Bouvardia ternifolia (Cav) Schl.), higuerilla (Ricinus communis L. 1753), escobilla (Gymnosperma glutinosum Cerv.) y mora (Morus alba L.). El repoblado se compone de fresnos jóvenes, pino (Pinus montezumae Lamb.), pirúl y algunos eucaliptos. En cuanto a la vegetación menor hay pasto blanco (Andropogon saccharoides Sw.), pasto búfalo (Buchloe dactyloides Nutt engelm), zacate amor (Eragrostis mexicana Link) y espiga negra (Hilaria cenchroides H.B.K).

Es la zona que mantiene la mejor condición actual de conservación en el PNMFN, encontrándose clasificada como "Buena", esto principalmente por la alta concentración de materia orgánica que presenta el suelo, el porcentaje de cobertura en la zona, la variación de especies vegetales, poseer algunos de los especímenes de mayor altura del PNMFN, el DN medido, entre otras características. Gran parte del área resulta de difícil acceso para los visitantes, por lo que el impacto que estos generan no es tan alto [4].

Zona 2: Estacionamiento principal. Tiene un área de 0,94 ha y suelos con profundidades de $0,35 \mathrm{~m}$ a 0,50 $\mathrm{m}$. La pedregosidad del suelo se clasifica como ligeramente pedregoso, existiendo además pocos afloramientos rocosos. La permeabilidad es moderada y el suelo se encuentra imperfectamente drenado. La zona se encuentra clasificada con erosión "moderada", principalmente por casos de erosión laminar. Un factor de degradación del suelo a considerar es la compactación, producto del uso que se le da a la zona. Aunque el área tiene como objetivo principal servir de estacionamiento de los visitantes, hay presencia relevante de eucaliptos (Eucaliptus camaldulensis Dehnh y Eucaliptus globulus Labill) y roble sedoso (Grevillea robusta A. Cunn.) que brindan sombra a automóviles y visitantes. También existe una muy pequeña área destinada a juegos infantiles que cuenta con una franja de casuarinas (Casuarina equisetifolia L.) que la separa de una zona más árida al norte. En el estra- to de vegetación menor es posible encontrar pasto búfalo (Buchloe dactyloides (Nutt endelm)) y navajita azul (Bouteloua gracilis (H.B.K.) Lag). En el sotobosque se encuentran principalmente moras (Morus alba L) y mejorana de campo (Brickellia veronicaefolia (H.B.K)), como repoblado casuarinas (Casuarina equisetifolia L.), eucaliptos (Eucaliptus camaldulensis Dehnh), pirúl (Schinus molle L.) y capulín (Prunus serotina Cav.).

El estado actual de conservación de la zona dos se clasificó como moderada, presenta algunos de los árboles de mayor porte y una buena cobertura arbórea $(31,9 \%)$, al igual que algunas áreas de la zona con un buen estado de vegetación y suelos. La erosión fue clasificada como moderada, teniendo el mayor impacto en las áreas descubiertas que son usadas como estacionamiento o zona de juegos.

Zona 3: Ruta de corredores. Se desplaza en ocho hectáreas con suelos arcillo arenosos, profundidad promedio de 0,3 m que la ubica como la segunda zona con menor profundidad dentro del parque. El suelo es ligeramente pedregoso con presencia moderada de afloramientos rocosos. La concentración de la materia orgánica es alta y el $\mathrm{pH}$ va de 7,5 a 8,5. No presenta problemas de erosión. Esta zona tiene una cobertura de dosel muy baja, la vegetación menor se compone principalmente por herbáceas como zacate amor (Eragrostis mexicana Link), pasto búfalo (Buchloe dactyloides Nutt engelm) y navajita azul (Bouteloua gracilis (H.B.K.) Lang). En el sotobosque se encuentra nopal (Opuntia streptacantha Lemaire), palo dulce (Eysenhardtia polystachya (Ort.)), Hierba del carbonero (Baccharis conferta Kunth) y uña de gato (Mimosa aculeaticarpa Hort.). En el repoblado es posible encontrar nopal (Opuntia streptacantha Lemaire) y algunos eucaliptos (Eucaliptus camaldulensis Dehnh y Eucaliptus globulus Labill) y pirules (Schinus molle L.). Finalmente, los pocos individuos de estrato arbóreo son eucaliptos, pirules y nopales (Opuntia streptacantha Lemaire). Los visitantes suelen utilizar esta zona como pista para correr o caminar.

Se clasificó como "deteriorada", a pesar de que la presión de los visitantes no es tan elevada, la falta de acciones de manejo ha promovido el deterioro constante del sitio. Muchos de los individuos arbóreos presentan daño por plaga o por incendio. La zona es una de las que presenta mayor cobertura herbácea $(89,4 \%)$. 
Zona 4: Ladera norte. Tiene un área de 5,18 ha. El suelo tiene profundidades que van de $0,10 \mathrm{~m}$ a más de $1,50 \mathrm{~m}$. La clase textural dominante es migajón arcillo arenosa, la estructura del suelo se encuentra débilmente desarrollada, la pedregosidad del suelo va de ligeramente pedregoso a muy pedregoso dependiendo del sitio de muestreo, presenta afloramientos rocosos. La permeabilidad es moderada, mientras que la clase de drenaje del suelo va de pobremente drenado a imperfectamente drenado. Baja presencia de raíces y poros en el suelo siendo en su mayoría finos o muy finos, para ambos casos. Se muestra "muy alta" concentración de materia orgánica en un suelo no calcáreo. El pH de la zona va de 7,16 a 8,3. La erosión es "Baja" debido a que no se encontraron señales de canalillos o cárcavas en los sitios medidos. La vegetación se compone en su mayoría por la vegetación menor de pasto búfalo (Buchloe dactyloides) y escobilla (Aristida adscensionis). El sotobosque está compuesto por especies como nopal (Opuntia streptacantha Lemaire), uña de gato (Mimosa aculeaticarpa Hort), mejorana de campo (Brickellia veronicaefolia (H.B.K)) y agave. El arbolado es escaso, compuesto por pirules (Schinus molle L.) y algunos eucaliptos y casuarinas. No se encontraron individuos de repoblado. Los individuos del estrato arbóreo encuentran dispersos en la zona, se encontrarón especies como acacia, eucalipto (Eucaliptus camaldulensis Dehnh), casuarina (Casuarina equisetifolia L.) y pirúl (Schinus molle L.), siendo este último el más abundante. La condición actual de conservación se clasificó como "deteriorada". El estrato arbóreo, además de tener una cobertura baja $(5,4 \%)$ presenta daño por plaga en la mayoría de los especímenes y un bajo diámetro normal.

Zona 5: Estacionamiento secundario. Tiene un área de 1,3 ha. La densidad de vegetación es muy baja debido a la alta presión que recibe por el tránsito de autos. Se observó una diferencia de profundidad en el suelo de tan solo $5 \mathrm{~cm}$ entre sitio y sitio. Presenta un suelo migajón arcillo arenoso, con estructura débilmente desarrollada. Hay poca incidencia de afloramientos rocosos y un porcentaje importante de pedregosidad (del 5 al 20\%). Existe una baja incidencia de poros y raíces, la materia orgánica es moderada y es un suelo ligeramente calcáreo. El pH promedio es de 8,3 , mientras que el rango de la zona va de 7,9 a 8,6. La erosión es moderada, encontrando principalmente señales de erosión laminar con una extensión de hasta $30 \mathrm{~m}$ de largo por $5 \mathrm{~m}$ de ancho. Es posible encontrar quintonil (Amarantus hybridus L.), acahual amarillo (Simsia amplexiacaulis Cav) y pasto búfalo (Buchloe dactyloides) como vegetación menor, prácticamente no existe sotobosque. Además, se tienen individuos de repoblado principalmente de pirúl (Schinus molle L.) y Thuja (Platycladus orientalis (L.) Franco). En cuanto al arbolado la especie dominante es el pirúl (Schinus molle L.). La profundidad del suelo es de 0,34 m que imposibilita el correcto desarrollo de especies arbóreas, repoblado y sotobosque. La zona tiene una clasificación de su condición actual de conservación de "deteriorada", debido a la baja presencia de arbolado y repoblado, aunado a la cobertura de pastos apenas está arriba del 50\%.

Zona 6: Área comercial y estacionamiento. No considerada en la evaluación.

Zona 7: Zona comercial. No considerada en la evaluación.

Zona 8: Planta de tratamiento/humedal. La zona posee un área de 0,51 ha siendo la más pequeña del parque. El suelo presenta dos clases texturales diferentes: arcillo arenoso y migajón arcillo arenoso. Se pudo identificar una estructura de suelo débilmente desarrollada. En uno de los sitios de muestreo se encontró afloramiento rocoso así como un porcentaje importante de pedregosidad (1 a 20\%). La permeabilidad va de lenta a moderada y se encuentra imperfectamente drenado en general. Existe una baja presencia de poros y raíces, clasificados como "pocos" para ambos casos. La materia orgánica se clasifica en la zona como "Moderada" y la aplicación de ácido clorhídrico muestra un suelo no calcáreo. El pH promedio es de 7,15 con rango que puede ir de 6,83 a 7,42. La erosión del suelo es moderada pero encontrando señales de erosión laminar. Alrededor de la zona crece pasto búfalo (Buchloe dactyloides (Nutt engelm)), en el sotobosque se puede encontrar mejorana de campo (Brickellia veronicaefolia (H.B.K)), tronadora(Tecoma stans H.B.K) y siempreviva (Sedum praealtum B), en el repoblado solo se encuentra nopal (Opuntia streptacantha Lemaire) y el estrato arbóreo se compone por yuca (Yuca filifera (Chabaud, 1976)), pirul (Schinus molle L.), casuarina y nopal (Opuntia streptacantha Lemaire).

La clasificación del estado actual de conservación de la zona es deteriorada. Los principales problemas son la pérdida de suelo por erosión y los daños que presentan los individuos del estrato arbóreo, la baja presencia de sotobosque y repoblado coadyuvan a 
que la zona mantenga esta clasificación, así como el daño presente en estos últimos.

Zona 9: Pastizal. Se refiere a un área de 1,82 ha y es una de las zonas de menor actividad por parte de los visitantes. En esta zona el suelo va de 10 a $60 \mathrm{~cm}$ y presenta textura migajón arcillo arenoso con una estructura moderadamente desarrollada. La incidencia de afloramientos rocosos va de pocos a medios, mientras que el porcentaje de pedregosidad va del 1 al $20 \%$. La zona posee una permeabilidad moderada y se encuentra imperfectamente drenada. Existe una baja presencia de poros y raíces para ambos casos. La materia orgánica se clasifica con contenido moderada, y es un suelo no calcáreo. El rango de $\mathrm{pH}$ va de 7,16 a 7,57. La pérdida de suelos por erosión es moderada, además de encontrar señales de compactación del suelo. Tiene poca pendiente del terreno y el estrato de vegetación menor es el dominante. En este se encuentra como especie dominante la escobilla (Aristida adscensionis L.), en el sotobosque hay uña de gato (Mimosa aculeaticarpa Hort), mejorana de campo (Brickellia veronicaefolia (H.B.K)) y nopal (Opuntia streptacantha Lemaire), el repoblado y el arbolado es con eucalipto (Eucaliptus camaldulensis Dehnh). Esta zona se clasificó en su condición actual de conservación como "deteriorada", ya que, a pesar de tener una cobertura herbácea del $65 \%$, existe una baja densidad de individuos de los estratos arbustivos y arbóreos, y los pocos individuos presentes de estos estratos presentan daños por agentes abióticos, fuego principalmente. La situación es similar para el poco repoblado existente, que además de eso enfrenta escasez de agua provocando que la supervivencia sea muy baja. La elevada cobertura de pastos aunada al poco o nulo manejo genera que sean un potencial combustible para incendios en la época de secas, generando daño a los individuos de otros estratos que logran establecerse.

Zona 10: Zona de convivencia y ruta equina. La zona posee un área de 3,02 ha, siendo una de las más concurridas del parque por los visitantes. Aquí se realizaron siete sitios de muestreo. Se observó suelos con profundidad promedio de $0,97 \mathrm{~m}$, con rango de 0,70 a $1,20 \mathrm{~m}$. La zona presenta un suelo migajón arcillo arenoso con estructura débilmente desarrollada, la incidencia de afloramientos rocosos es baja y presenta pedregosidad cambiante (desde 1 y hasta $75 \%)$. La zona posee una permeabilidad moderada y se encuentra imperfectamente drenado en general.
Hay baja presencia de materia orgánica en un suelo no calcáreo. El rango de pH va de 7,31 a 8,2. La erosión medida en la zona se encuentra clasificada como "alta", debido a que el mal manejo del sitio y las actividades que se desarrollan han contribuido a acentuar algunas áreas que presentan señales de erosión hídrica. Se observó compactación del suelo y degradación físico - química. Los recorridos a caballos y los residuos sólidos que ahí se generan producen mayor presión en la zona. A pesar de esto, el hecho de que el rio atraviese la zona favorece a la vegetación. Es posible encontrar pasto blanco (Andropogon saccharoides Sw.), espiga negra (Hilaria cenchroides H.B.K.) y pasto búfalo (Buchloe dactyloides (Nutt engelm)) como vegetación menor, el sotobosque se compone por cabello de ángel (Calliandra grandofilia (L'Hér.) Benth) y capulín (Prunus serótina Cav.). El repoblado por eucaliptos (Eucaliptus camaldulensis Dehnh), fresnos (Fraxinus udhei (Wenz.) Lingelsh), roble sedoso (Grevillea robusta A. Cunn.), Acacia (Acacia retinoides Schltdl.), thuja (Platycladus orientalis (L.) Franco), pirúl (Schinus molle L.), tepozán (Buddleia cordata H.B.K.) y cedro blanco (Cupressus lindleyi Klotzsch ex Endl). Por último, en el arbolado se encuentra encino, tepozán (Buddleia cordata H.B.K.), fresno (Fraxinus udhei (Wenz) Lingelsh)), eucalipto (Eucaliptus globulus Labill y Eucaliptus camaldulensis Dehnh), ahuehuetes ( $\mathrm{Ta}$ xodium mucronatum Ten.) y acacia (Acacia retinoides Schltdl.). La zona se calificó con condición moderada en su estado de conservación, aunque es una de las que posee los arboles de mayor altura, diámetro normal y mayor variación de especies. Se encuentra bajo presión constante de los visitantes dado que las actividades que ahí se realizan han generado un daño extendido a la mayoría de los individuos; la erosión del suelo es alta y existen evidencias de compactación del suelo en distintas áreas.

Zona 11: Cascada. La zona se refiere a una extensión de 0,87 ha y se encuentra al pie de la zona comercial donde la incidencia de visitantes es relativamente baja la mayor parte de la semana. Aquí se observaron profundidades que van de $50 \mathrm{~cm}$ a $90 \mathrm{~cm}$. El suelo es de textura arena migajosa sin estructura aparente. La presencia de afloramientos rocosos es alta y el porcentaje de pedregosidad resulta ser uno de los mal altos del parque. La permeabilidad del suelo resulta ser rápida y se encuentra bien drenado. Existe una baja presencia de poros y raíces, y la materia orgánica es baja. El pH va de 6,98 a 7,67. La erosión se clasifica como media, siendo uno de los tres sitios 
evaluados el que concentra los rasgos más pronunciados de erosión. Crecen especies herbáceas como el pasto blanco (Andropogon saccharoides Sw), trébol y la navajita azul (Bouteloua gracilis (H.B.K.) Lag), el sotobosque se compone de palo dulce (Eysenhardtia polystachya (Ort.)), mora (Morus alba L.) y hierba del carbonero (Baccharis conferta Kunth). Como repoblado se encuentra cedro blanco (Cupressus lindleyi Klotzsh ex Endl.), pirúl (Schinus molle L.), tejocote (Crateagus pubescens (Kunth) Steud) y fresno (Fraxinus udhei (Wenz.) Lingelsh). Por último, como arbolado crece pirul (Schinus molle L), aile (Alnus acuminata H.B.K.), tejocote (Crateagus pubescens (Kunth) Steud), ahuehuete (Taxodium mucronatum Ten.) y fresno (Fraxinus udhei (Wenz.) Lingelsh). La zona se ubicó como moderadamente conservada, dado el desarrollo de vegetación arbórea, así como la reducción del espacio que se generó con la construcción de la ex hacienda y la baja presencia de materia orgánica en el suelo.

Zona 12: Vivero CONAFOR. La zona es un área de 9,18 ha de extensión y está administrada por CONAFOR. Es utilizada para la producción de árboles en vivero. La profundidad del suelo va de $1,6 \mathrm{~m}$ a 2,1 m. El suelo es de textura migajón arenoso, con una estructura débilmente desarrollada y pedregosidad que va del 1 al 20\%. La zona posee permeabilidad moderada y es imperfectamente drenado. Existe baja presencia de materia orgánica en un suelo que se clasifica como ligeramente calcáreo. El pH promedio es de 7,91. La erosión se clasifica como baja debido a las constantes actividades realizadas para la conservación del suelo, y las actividades de nivelación para el establecimiento del vivero y las especies a producir. La vegetación menor se compone por pasto búfalo (Buchloe dactyloides (Nutt engelm)) principalmente y el arbolado por eucaliptos (Eucaliptus camaldulensis Dehnh) y pirules (Schinus molle L.).

Zona 13: Zona concesionada. La zona es de un área de 7,69 ha y la profundidad de suelo que van de 60 a $90 \mathrm{~cm}$. El suelo es de textura migajón arcillo arenosa con estructura que va de débilmente desarrollada a moderadamente desarrollada. No se encuentran afloramientos rocosos y se clasifica como ligeramente pedregoso. El suelo es imperfectamente drenado con permeabilidad moderada. Existe baja presencia de poros y raíces. La materia orgánica es moderada y es de tipo no calcáreo. El rango de $\mathrm{pH}$ va de 6,46 a 7,72 . La pérdida de suelos por erosión es baja ya que es una de las áreas menos perturbadas del parque, aunque es posible encontrar algunas zonas compactadas. Es la segunda zona de mayor superficie, la vegetación menor se compone por pastos caducifolios como el pasto búfalo (Buchloe dactyloides (Nutt engelm)) y la espiga negra (Hilaria cenchroides H.B.K.), el sotobosque por uña de gato (Mimosa aculeaticarpa Hort), escobilla (Aristida adscensionis L.) y hierba del carbonero (Baccharis conferta Kunth), el repoblado por eucaliptos (Ecualiptus camaldulensis D.) en su mayoría y algunos cedros blancos (Cupressus lindleyi Klotzsch ex Endl.) y pirules (Schinus molle L.) en la parte más alta. En el estrato arbóreo se observa un gran porcentaje de eucaliptos (Eucaliptus camaldulensis Dehnh) y algunos pirules (Schinus molle L.) y nopales (Opuntia streptacantha Lemaire). La zona se catalogó con condición de moderadamente conservada, es la zona más alejada de parque y la incidencia de visitantes es casi nula. Sin embargo, posee una cobertura de arbolado baja, apenas un $8,60 \%$, los cuales tienen un vigor moderado y una altura pobre con relación a los individuos de algunas otras zonas.

Zona 14: Casco de hacienda. No considerada en la evaluación.

\section{El estado actual de conservación del PNMFN}

De las 14 zonas de manejo se calificaron 11 , y de estas las de mayor deterioro son cinco: 3, 4, 5, 8 y 9 que representan el 34\% del PNMFN. La problemática recurrente es la falta de manejo y reglamentación, que genera problemas tanto al suelo como a la vegetación. En el caso de la vegetación se puede mencionar el daño por plaga, daño por incendio, diversos problemas en su desarrollo, como: porte pobre, alturas que se encuentran por debajo del promedio, inviabilidad del repoblado, daño generado por visitantes, entre otros. Aunado a esto la falta de agua incrementa las dificultades de supervivencia de las especies vegetales.

En lo que se refiere a un grado de conservación media se tiene a cuatro zonas: 2, 10, 11, y 13 para el $25 \%$ de la superficie del PNMFN. La principal característica que tienen en común es que mantienen un buen nivel de cobertura de vegetación, aunque ciertas zonas e individuos presentan problemas de alguna índole, e incluso la zona presenta áreas con niveles de degradación en suelos y vegetación importantes.

La zona que resultó con la mejor conservación fue la 1 que representa el $11 \%$ del parque. Se debe princi- 
palmente a que las condiciones de suelo y agua resultan benéficas para una gran variedad de especies, además de que él porcentaje de visitantes no es tan alto como para generar el mismo impacto generado a otras zonas del PNMFN que se encuentran cercanas al área comercial y el casco de la hacienda, especialmente en la ribera del río. Conocer el estado actual de los recursos naturales permite programar y presupuestar los recursos financieros, humanos y materiales para su conservación, mejoramiento e incluso fundamentar su manejo [10, 23].

\section{El Estado del Río Coxcacuaco}

La caracterización a lo largo del río consistió en 4 puntos de muestreo previamente indicados y descritos a continuación:

Punto 1. En este punto el río ya ha recibido descargas de aguas residuales de algunas poblaciones pero los factores contaminantes más significativos aun no lo afectan. Se encontró un aumento en el pH llegando hasta 7,1. La conductividad eléctrica llega casi a los 700 4s, los sólidos totales se mantienen entre los 525 y $995 \mathrm{mg} / \mathrm{L}$, con excepción del sábado por la mañana, cuando llegan hasta $15635 \mathrm{mg} / \mathrm{L}$. La turbidez alcanza 70 UNF y la alcalinidad de $\mathrm{HCO}_{3}$ es la predominante. La cantidad de cloruros es baja y la dureza total se considera alta, llegando hasta 350 $\mathrm{mg} / \mathrm{L}$. La DBO presentó su máximo en $60,8 \mathrm{mg} / \mathrm{L}$.

Punto 2. El punto de muestreo se ubicó después de donde las fuentes del ambiente generativo y degenerativo se incorporan, mucho delante del punto 1 . Las características organolépticas son poco favorables, el $\mathrm{pH}$ bajó considerablemente y la conductividad eléctrica llegó casi a los 1001 4s. Los sólidos totales aumentaron drásticamente con respecto al primer punto, siendo el valor más bajo de 2550 y el más alto de $8850 \mathrm{mg} / \mathrm{L}$. La turbidez alcanzó casi 700 UNF y de nuevo la alcalinidad de $\mathrm{HCO}_{3}$ es la que predominó. La cantidad de cloruros fue baja y la dureza total se consideró alta, ligeramente menor que en el punto anterior al llegar hasta los 262 mg/L. La DBO5 llegó a su máximo con $328 \mathrm{mg} / \mathrm{L}$.

Punto 3. El lugar se seleccionó donde se desecha el agua que los comercios establecidos dentro del parque vierten. El pH se mantiene entre 6,7 y 7,2, y la conductividad eléctrica superó a los 1050 4s. Los sólidos totales disminuyeron ligeramente con respecto al punto 2 , siendo el valor más bajo de 985 y el más alto de casi 2600 mg/L. La turbidez alcanza 195 UNF y la alcalinidad de $\mathrm{HCO}_{3}$ es la que predomina, la cantidad de cloruros se consideró baja y la dureza total llegó hasta los $275 \mathrm{mg} / \mathrm{L}$. La DBO5 llegó a su máximo valor con $170 \mathrm{mg} / \mathrm{L}$. Las características organolépticas son poco favorables al igual que en los anteriores puntos.

Punto 4. Se encuentra hacia la salida del polígono del parque aparentemente ya no existen descargas de aguas residuales. Las características organolépticas de nuevo no son favorables, el $\mathrm{pH}$ fue estable, manteniéndose entre 6,8 y 7,2, la conductividad eléctrica disminuyó ligeramente y no superó los 866 4s, Los sólidos totales alcanzaron un valor hasta de 2270 $\mathrm{mg} / \mathrm{L}$, la turbidez en cambio no superó las 47 UNF y la alcalinidad de $\mathrm{HCO}_{3}$ es la que predomina, la dureza total se ubicó entre 150 y $225 \mathrm{mg} / \mathrm{L}$ y es considerada alta. La DBO5 llega a su máximo valor con $36 \mathrm{mg} / \mathrm{L}$.

Los resultados obtenidos en laboratorio se compararon con las normas de calidad de agua de referencia (NOM127, SSA1994, OMS, NOM001-ECOL1996), casi todos los parámetros muestran que la calidad de agua rebasa los niveles permitidos y la ubicación de los puntos de muestreo permitió identificar y comprobar las fuentes de contaminación para el río.

El pH solamente infringe la norma en solo un momento del muestreo, con un valor de 6,4. La baja variabilidad en el $\mathrm{pH}$ indica que el rio no está recibiendo descargas de desechos industriales. La conductividad eléctrica puede indicar contenido de sólidos disueltos, entre ellos sales en el agua, en todos los momentos de muestreo se rebasa la norma. Esto puede deberse a que la pendiente disminuye en este tramo, y los sólidos (en este caso solubles) se concentran aquí. Los sólidos no solubles tienden a sedimentarse, por lo que el comportamiento de este parámetro alcanza su valor máximo en el punto 2 y de inmediato comienza una disminución, ya que se acumulan en el fondo por el estancamiento del agua. El agua también se encuentra con la máxima turbiedad en el punto 2 y el máximo valor de DBO. Los sólidos, la turbidez y la DBO5, tienen como fuente los desagües vertidos de aguas residuales y acumulación de partículas producto de la erosión; los efectos incluyen la reducción de la efectividad en el tratamiento del agua; el desprendimiento de magnesio y hierro favorecen la presencia de amoniaco. La alcalinidad al $\mathrm{HCO}_{3}$ y por los valores de dureza se puede clasificar 
esta agua como muy blanda. La cantidad de cloruros no rebasa el límite permisible.

Habiendo identificado que el punto dos resultó ser el más contaminado es posible definir que el proceso de tratamiento de las aguas residuales tanto de las comunidades cuenca arriba y del reclusorio no son los más adecuados, no están siendo eficientes y eficaces. Lo anterior es importante para considerar riesgos como la pérdida de hábitat o cambio climático [24]ecological niche models (ENMs.

\section{CONCLUSIONES}

Los resultados muestran deterioro en las áreas zonificadas dentro del parque nacional Molino de Flores Netzahualcóyotl, con mayor impacto en aquellas con mayor presencia de visitantes. La condición actual de los recursos naturales del parque muestra la urgencia en implementar acciones que restauren los ciclos ecológicos deteriorados, así como poner en marcha políticas y prácticas en pro de la conservación del medio natural. A veinte años de ser administrado por el municipio de Texcoco la conservación de los recursos parecen venir a la baja mientras que la presión por el turismo y comerciantes al alza. Ante el evidente deterioro resulta necesario promover la participación de autoridades, comerciantes y visitantes en cualquier plan de acción que se pretenda llevar a cabo por parte de la administración. Debe considerarse la elaboración participativa de un programa de manejo del parque.

\section{AGRADECIMIENTOS}

A la Universidad Autónoma Chapingo a través del Centro de Investigación en Recursos Naturales y Medio Ambiente (CIRENAM). Se agradece a los revisores anónimos que mejoraron el manuscrito.

\section{REFERENCIAS}

[1] TOLEDO, D., SANDERSON, M., JOHNSON, H., REEVES, J. L., DERNER, J. D., VERMEIRE, L., and HENDRICKSON, J. Evaluating plant biodiversity measurements and exotic species detection in $\mathrm{Na}$ tional Resources Inventory Sampling protocols using examples from the Northern Great Plains of the
USA. Ecological Indicators, 46, 2014, p. 149-155 https://doi.org/10.1016/j.ecolind.2014.06.020

[2] PINEDA-LÓPEZ, M.R., RUELAS I. E., SÁNCHEZ V. L., ESPINOZA G.M., ROJO A.A. y VÁSQUEZ M.S. Dinámica de uso y cobertura del suelo en un parque nacional mexicano. Madera y Bosques, 23(3), 2017, p. 87-99, https://doi.org/10.21829/ myb.2017.233149

[3] LGEEPA. Ley general de equilibrio ecológico y protección al ambiente. Diario Oficial de la Federación DOF enero 28, 1988. Gobierno de la República. México. 135 p., http://www.diputados.gob.mx/LeyesBiblio/ref/lgeepa.htm

[4] GLYNN, P.D., VOINOV, A.A, SHAPIRO, C.D. and WHITE, P.A. From data to decisions: Processing information, biases, and beliefs for improved management of natural resources and environments. Earth's Future, 5(4), 2017, p. 356-378

[5] RAMÍREZ, A. Plan de Restauración Ecológica para el Área de Conservación del Parque Nacional Molino de Flores [Tesis de maestría]. Texcoco (México): Universidad Autónoma Chapingo, Departamento de Suelos, 2013, 118 p.

[6] LEYVA-OVALLE, Á., VALDEZ-LAZALDE, J., DE LOS SANTOS-POSADAS, H., MARTÍNEZ-TRINIDAD, T., HERRERA CORREDOR, J., LUGO-ESPINOSA O., y GARCÍA-NAVA J. Monitoreo de la degradación forestal en México con base en el inventario nacional forestal y de suelos (INFyS). Madera y Bosques, 23(2), 2017, p. 69-83, http://dx.doi.org/10.21829/ myb.2017.2321431

[7] ROSETE-VERGÉS, F.A., JL PÉREZ-DAMIÁN, M VILLALOBOS-DELGADO, E.N. NAVARRO-SALAS, E. SALINAS-CHÁVEZ y R. RERNOND-NOA. El avance de la deforestación en México 1976-2007. Madera y Bosques, 20(1), 2014, p. 21-35

[8] FERNÁNDEZ-LANDERO, A.,y VAZQUEZ-GARCÍA, D. Diagnóstico integral de conservación del parque nacional Molino de Flores, Texcoco [Tesis de maestría en conservación y restauración de bienes culturales]. Ciudad de México (México): Instituto Nacional de Antropología e Historia, 2014, 264 p.

[9] DÁVALOS, R. El papel de la investigación científica en la creación de las áreas naturales protegidas. Madera y Bosques, 22(1), 2016, p. 7-13.

[10] BENAVIDES, H., y FERNÁNDEZ D. Estructura del arbolado y caracterización dasométrica de la segunda sección del Bosque de Chapultepec. Madera Bosques, 18(2), 2012, p. 51-71. 
[11] MAGUELLAN. MobileMapper 6. Guía básica de utilización. Santa Clara (USA): 2008 p. 30

[12] SUN, L., y ZESHENG Z. Modelling yield of water hyacinth (Eichhornia crassipes) using satellite and GPS sensors. 6th International Conference on Agro-Geoinformatics, 2017, p. 1-5

[13] HANNAH, L., DONATTI, C., HARVEY, C., ALFARO, E., RODRIGUEZ, D., BOURONCLE, C., CASTELLANOS, E., DIAZ, F., FUNG, E., HIDALGO, H., IMBACH, P., LÄDERACH, P., LANDRUM J., and SOLANO A. Regional modeling of climate change impacts on smallholder agriculture and ecosystems in Central America. Climatic Change, 41, 2016, p. 29-45, https://doi.org/10.1007/s10584-016-1867-y

[14] MÉXICO. COMISIÓN NACIONAL FORESTAL. Inventario Nacional Forestal y de Suelos. Procedimiento de muestreo. Guadalajara (México): CONAFOR, 2015, p. 261

[15] ESTRADA-HERRERA, I., HIDALGO-MORENO, C., GUZMÁN-PLAZOLA, R., ALMARAZ-SUÁREZ, J., NAVARRO-GARZA H., y ETCHEVERS-BARRA J. Indicadores de calidad de suelo para evaluar su fertilidad. Agrociencia, 51(8), 2017, p. 813-831

[16] CHAMIZO, S., RODRÍGUEZ-CABALLERO, E., ASENSIO C., and DOMINGO, F. Penetration resistance of biological soil crusts and its dynamics after crust removal: relationships with runoff and soil detachment. Catena, 126, 2016, p. 164-172

[17] MIRALLES, I., ORTEGA, R., CANTÓN Y., and ASEN$\mathrm{SIO}, \mathrm{C}$. Soil degradation from excess salt and its relation to the topography on an area of Southern Spain. Agrochimica, 46(6), 2002, p. 270-279

[18] VÁZQUEZ-MARTÍNEZ, G. y VÁZQUEZ-SOLÍS V. Evaluación de recursos naturales y culturales para la creación de un corredor turístico en el altiplano de San Luis Potosí, México. Investigaciones Geográficas, (94), 2017, p. 1-15

[19] FRANCH-PARDO, I. y CANCER-POMAR, L. EI componente visual en la cartografía del paisaje. Aptitud paisajística para la protección en la cuenca del río Chiquito (Morelia, Michoacán). Investigaciones Geográficas, 93, 2017, p. 42-60

[20] ARRIAGA-VÁZQUEZ, A. Estado Actual y Propuestas de Restauración del Río Coxcacuaco [Tesis de licenciatura]. Texcoco (México): Universidad Autónoma Chapingo, Departamento de Suelos, 2012, 93 p.

[21] VÁZQUEZ-ALARCON, A. Manual de prácticas del curso de contaminación ambiental. 1 ed. Chapingo (México): Universitaria, 2017, 75p.
[22] PAREDES-GONZALEZ, A., MONTERROSO-RIVAS, A., RODRÍGUEZ-ESPARZA L. and ZAMUDIO-SÁNCHEZ F. Projection and probability of land use change in Zoquiapan, Mexico: considerations for forest management. Revista Chapingo, Serie Ciencias Forestales y del Ambiente, 24(1), 2018, p. 59-71.

[23] GÓMEZ-DÍAZ, J.D., MONTERROSO-RIVAS, A.I., LECHUGA-GAYOSSO, L., ARCE-ROMERO A.R., and RUIZ-GARCIA P. Impact of Climate Change on Soil Organic Carbon Content on Agricultural Soils of Mexico. Advances in Intelligent Systems and Computing, 2018. p. 58-69. doi:10.1007/9783-319-70187-5_5

[24] PRIETO-TORRES, D.A., NAVARRO-SIGÜENZA, A., SANTIAGO-ALARCON D. and ROJAS-SOTO O. Response of the endangered tropical dry forests to climate change and the role of Mexican Protected Areas for their conservation. Global Change Biology, 2016, 22(1), 364-379. http://doi.org/10.1111/gcb.13090 